\title{
A new local anaesthesia technique for cataract extraction by one quadrant sub-Tenon's infiltration
}

\author{
J D Stevens
}

\begin{abstract}
A new technique of local anaesthetic administration has been used for $\mathbf{5 0}$ patients undergoing cataract extraction. The simple technique involves direct transconjunctival infiltration of local anaesthetic directly to the sub-Tenon's space, in the inferior-nasal quadrant, using a blunt 19-gauge Southampton cannula. This method seeks to avoid the risks of retrobulbar haemorrhage, perforation of the globe, damage to the optic nerve, and injection into the subarachnoid space, whilst providing prolonged and reliable anaesthesia. Akinesia is achieved by the inferior-nasal placement of solution and if not sufficient, a top-up can easily be given. Patients graded any discomfort or pain using a $10 \mathrm{~cm}$ visual analogue graphical pain score chart with numerical and descriptive rating scale. The delivery of 50:50 mixture of lignocaine $2 \%$ and bupivacaine $0.5 \%$ anaesthetic was evaluated by patients with a median response of 'slight discomfort'. The operative procedure was graded with a median of 'no pain or discomfort', both for extracapsular cataract extraction and phakoemulsification. This is a new, modified, sub-Tenon technique which is simple, reliable, and which offers excellent anaesthesia and akinesia and avoids a sharp instrument being passed into the orbit.
\end{abstract}

(BrF Ophthalmol 1992; 76: 670-674)

In 1884 Knapp reported successful cataract extraction by topical anaesthesia using frequent drops of $5 \%$ cocaine. He commented on the need for more extensive anaesthesia if the iris is to be anaesthetised. Describing a technique for enucleation, he reported the successful use of a retrobulbar injection, ${ }^{1}$ rotating the globe nasally and introducing a needle through the muscle cone temporally. In 1884 Turnbull also described a local anaesthetic technique for enucleation, where topical $4 \%$ cocaine was applied to the cornea and conjunctiva, followed by the opening of Tenon's fascia and cocaine being dropped into the cut. ${ }^{2}$ Blunt pointed scissors which were curved on the flat were introduced from the nasal side and drops instilled. This was reported as successful in causing anaesthesia and vasoconstriction.

Today various methods of local anaesthesia are in use for cataract extraction including retrobulbar, ${ }^{3}$ peribulbar, ${ }^{4}$ subconjunctival, ${ }^{5}$ and subTenon's ${ }^{6}$ application of local anaesthetic solution. The desire to find an alternative to retrobulbar anaesthesia has been caused by a variety of unusual but potentially severe complications arising with this technique, ${ }^{78}$ including globe perforation, retrobulbar haemorrhage, and accidental injection of solution into a blood vessel or the subarachnoid space.

Peribulbar anaesthesia has been advocated to reduce risk of respiratory arrest and other systemic complications associated with retrobulbar anaesthesia and since the muscle cone is not entered, local anaesthetic solution is delivered away from the globe, so minimising risk to the eye. Anaesthesia is achieved by diffusion, often requiring a greater volume of solution than for true intraconal retrobulbar anaesthesia. In spite of an injection positioned away from the globe, peribulbar anaesthesia has been reported to be associated with serious complications, including globe perforation..$^{10}$

For both retrobulbar and peribulbar techniques a sharp, or relatively sharp, needle is 'blindly' placed into the orbit to deliver the anaesthetic solution. To avoid injecting deep into the orbit subconjunctival anaesthesia has been advocated, involving a subconjunctival injection of anaesthetic at the 12 o'clock position ${ }^{5112}$; this technique also being termed limbal subconjunctival injection. ${ }^{13}$ In a cooperative patient able to maintain ocular position, topical and subconjunctival anaesthesia have been shown to be effective in providing anaesthesia though more conjunctival chemosis occurs with subconjunctival than with retrobulbar or peribulbar anaesthesia. ${ }^{14}$ Many ophthalmic surgeons are reluctant to adopt a technique that does not provide sufficient ocular akinesia and in patients less able to understand the requirements of the ophthalmologist, or who are anxious and unable to communicate properly, general anaesthesia or a technique involving good akinesia is desirable. Residual ocular muscle function not only allows unexpected globe movement during surgery but may increase intraocular pressure.

Direct sub-Tenon's delivery of solution using a blunt cannula offers an alternative method. For retinal surgery ${ }^{15}$ and for cataract extraction ${ }^{6}$ a technique has been described where the conjunctiva is anaesthetised by subconjunctival injection of local anaesthetic solution and then Tenon's fascia is opened using blunt Westcott scissors. A blunt 19 gauge cannula directly irrigates solution posterior to the globe. In the case of retinal surgery, solution is delivered to all four quadrants $s^{5}$ and for cataract surgery ${ }^{6}$ the superior quadrants have been used.

A simple technique that produces excellent anaesthesia and reliable akinesia, with minimal risk of perforation of the globe, is the ideal. This paper describes a single quadrant inferior-nasal sub-Tenon's approach with a blunt cannula which is a modification of the original idea of Turnbull, ${ }^{2}$ the four quadrant method of Mein and Woodcock, ${ }^{15}$ and the superior quadrant method of Hansen et al. ${ }^{6}$ It has been developed to
City Road, Eye Hospital, 2PD

J D Stevens

Accepted for publication

8 June 1992 
be an effective and minimal risk anaesthetic technique for cataract surgery.

\section{Method}

Fifty patients who underwent cataract extraction with posterior chamber lens implantation who were scheduled for local anaesthesia, had an anaesthetic blockade by direct blunt cannula sub-Tenon's delivery. After informed consent, in the holding bay within the operating theatre suite, four drops of topical amethocaine $1 \%$ were applied to the cornea and conjunctiva followed by one drop of adrenaline $0 \cdot 1 \%$. A blunt Westcott (spring) scissors was taken and a small 'nick' incision made in the conjunctiva in the inferiornasal quadrant approximately $5 \mathrm{~mm}$ from the limbus (Fig 1). A blunt 19 gauge Southampton curved cannula was used to deliver a small bleb of local anaesthesia, using a 50:50 mixture of lignocaine $2 \%$ and bupivacaine $0.5 \%$ without adrenaline. This facilitates identification and elevation of Tenon's fascia and fine underlying intermuscular septal fascia. The Wescott scissors are then slid through the nick in the conjunctiva and used to create another 'nick' taking care to create a path in Tenon's capsule and the intermuscular septal fascia. Moorfields forceps are used to grip the conjunctival edge where the small nick was made and the curved cannula is then inserted onto bare sclera and glided along a path following the contour of the globe, until posterior to the equator (Fig 2). It is very important to pass through conjunctiva, Tenon's fascia, and the intermuscular septum, to reach bare sclera and therefore into the Tenon's space, before sliding the cannula posteriorly. Often, after the cannula is passed back in the potential space between the intermuscular septum, Tenon's, and sclera, a resistance is felt owing to fascia adherent to sclera posterior to the equator. This tissue may be passed through by application of gentle pressure to the syringe and after a few seconds a slight 'give' is felt, solution begins to flow, and the cannula may be further advanced. After delivery of approximately $1 \mathrm{ml}$, at this level, just posterior to the equator, the cannula is advanced further to a total distance of approximately 1.5 to $2.0 \mathrm{~cm}$, depending upon globe size,

Figure 1 Ablunt Westcott (spring) scissors is taken and a small 'nick' incision made in the conjunctiva in the inferonasal quadrant approximately $5 \mathrm{~mm}$ from the limbus. A blunt 19 gauge Southampton curved cannula is used to deliver a small bleb of local anaesthesia, so raising Tenon's capsule allowing better visualisation.

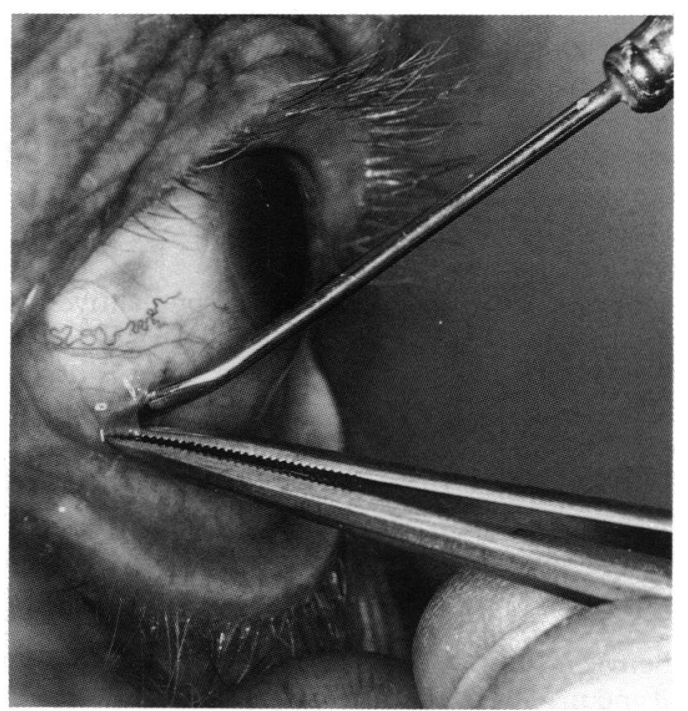

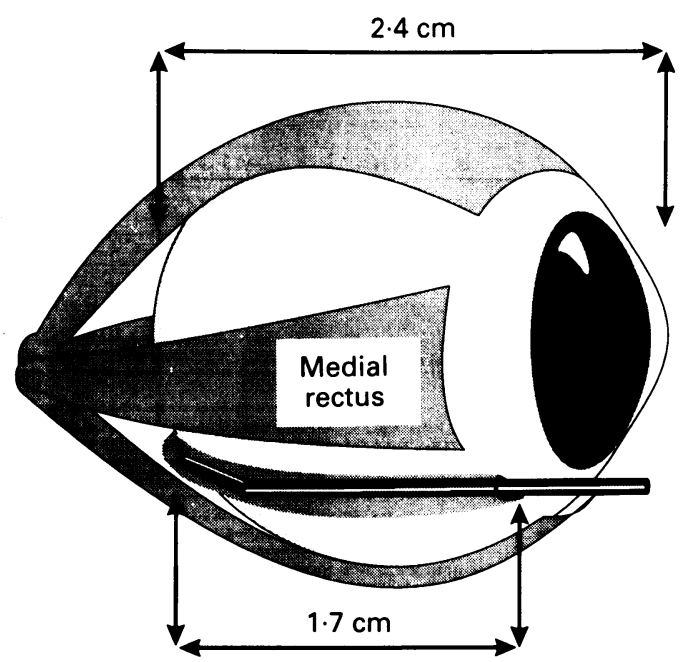

Figure 2 Schematic diagram of the path of the Southampton cannula, following the contour of the globe, to deliver anaesthetic solution posterior to the equator.

and further solution delivered to a total volume of 3.0 to $3.5 \mathrm{ml}$. At this stage a small degree of proptosis of the globe is usually visible, this providing confirmation that the solution has been delivered to the correct place. If care is not taken to deliver solution to the posterior subTenon potential space, then it is very easy to deliver anaesthetic solution as an anterior subconjunctival application. Immediate conjunctival chemosis then results and this may be extensive if the whole volume is given anteriorly by mistake. It is important therefore for the volume to be given slowly and in graded steps, so ensuring that the site of delivery is that which was intended. With practice, the cannular position, with respect to the fascial layers, can be judged accurately by the operator and the technique becomes quicker and easier.

After giving the anaesthetic solution at least 15 minutes was allowed for diffusion to occur before commencing surgery. Immediately prior to surgery, assessment of the amount of akinesia, presence and degree of ptosis, and eyelid tone was made. Akinesia was assessed by a nonmasked, four category, subjective scale graded as complete movement remaining, moderate movement, slight movement, no movement. If the eyelids were not flaccid immediately prior to surgery, an additional Van Lint facial block was given.

Each patient was shown a $10 \mathrm{~cm}$ visual analogue graphic pain score chart, on the first postoperative day, with numerical and descriptive rating scale, graded from 0 to 10 (Fig 3). Patients were shown the chart and invited to score where on the chart they could grade any discomfort or pain experienced. If patients were unable to see the chart, or read the accompanying text (printed at N10 size), a verbal explanation and description of the scoring chart was performed. Patients were first requested to score verbally any pain or discomfort for the delivery of the anaesthetic and then separately score the operation itself.

\section{Anatomy}

Connecting the extraocular muscle capsules is a sheet of condensed fascial tissue, the inter- 


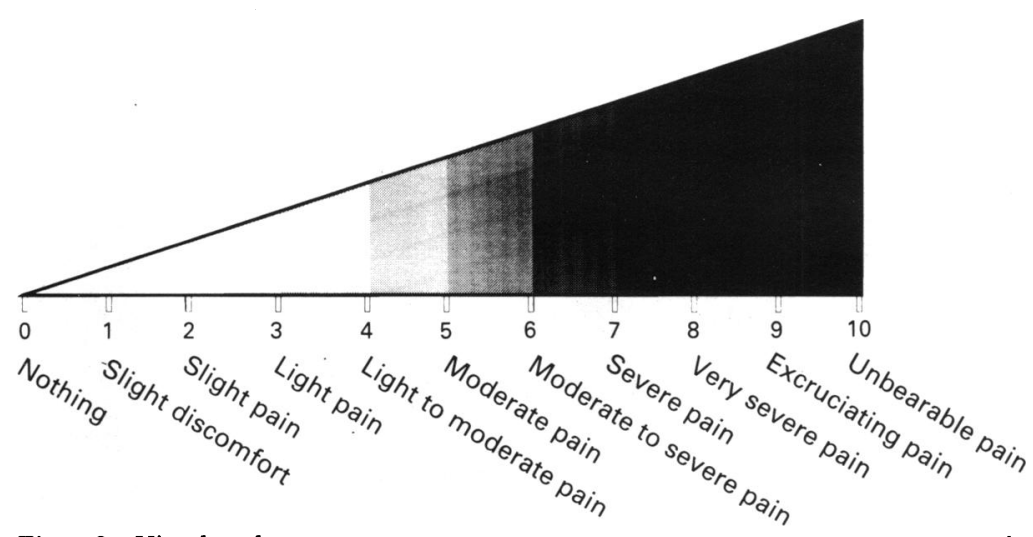

Figure 3 Visual analogue graphic pain score chart. A $10 \mathrm{~cm}$ pain score chart with numerical and descriptive rating scale.

muscular septum. As the extraocular muscles pass forward they increase in separation from each other, but the intermuscular septum continues to connect them. External to the extraocular muscles is a fascial plane, the Tenon's capsule. ${ }^{16}$ Tenon's capsule is a relatively dense fascial layer of elastic connective tissue surrounding the globe and extraocular muscles extending from the limbus to the optic nerve. All the extraocular muscles penetrate Tenon's capsule, the four recti penetrating posterior to the equator. Tenon's capsule anterior to this has been termed the anterior portion and extends to the limbus. A posterior portion of Tenon's capsule extends from the rectus penetrations back to the optic nerve and is thinner. The inner surface of the posterior portion of Tenon's capsule apposes sclera as there is no sub-Tenon's intermuscular septum present. A potential space exists between sclera and Tenon's capsule, the Tenon's or episcleral space, which does not normally contain any fluid. The anterior Tenon's capsule and sclera are separated by the intermuscular septum which extends between the extraocular muscle capsules in the same way as between the muscles in the posterior orbit. At about $3 \mathrm{~mm}$ from the limbus the anterior Tenon's capsule and sub-Tenon's intermuscular septum fuse to form a single fascial plane which further fuses with conjunctiva $1 \mathrm{~mm}$ from the limbus. Incision further back than $3 \mathrm{~mm}$ from the limbus must pass through all three layers. Incising conjunctiva closer than $3 \mathrm{~mm}$ from the limbus allows one incision to pass through conjunctiva, Tenon's capsule, and the intermuscular septum simultaneously. This would be an advantage to easily reach bare sclera, but by cutting the conjunctiva further back at approximately $5 \mathrm{~mm}$ from the limbus, less of a curve is required for the path of the cannula as it follows the contour of the globe. There is less trauma to the conjunctiva with a direct posterior path, with less risk of conjunctival tearing.

Tenon's space delivery allows potential diffusion of the solution throughout this compartment, and solution may also diffuse back into the orbit, reaching the intraconal central region. Koorneef has stated that there is no clear cut compartmentalisation into central (retrobulbar) region and peripheral space outside the muscle cone, not finding a common muscle sheath by serial section study. ${ }^{17}$ Solution may then diffuse extensively between compartments if sufficient time is allowed and may be facilitated by use of hyaluronidase.$^{18}$ Hyaluronidase has not been used in this study owing to difficulties in supply during the period of the study, though when available it would be anticipated that its use may enhance the efficacy of the technique.

To eliminate pain sensation for the globe, pain fibres exiting from the eye must be blocked and to further achieve akinesia, the extraocular muscle nerve supply should also be inhibited. Ocular pain sensation is mediated by the long sensory root of the ciliary ganglion. These fibres pass through the ganglion and provide sensation from the cornea, iris, and ciliary body. ${ }^{18}$ The ciliary ganglion is situated posteriorly in the orbit approximately $1 \mathrm{~cm}$ anterior to the optic foramen between the optic nerve and the lateral rectus muscle within the muscle cone. It is separated from the lateral rectus muscle by loose fat and is close to the ophthalmic artery. Posterior diffusion of local anaesthetic solution may directly block the ganglion, or blockade of muscle function may occur by solution affecting nerves passing more anteriorly. The differential temporal decrease in muscle function, with those muscles closest to the site of solution delivery, medial and inferior rectus being lost first, suggests that a direct block upon anterior nerve fibres may be occurring.

The extraocular muscles are supplied by cranial nerves III, IV, and VI. To achieve akinesia, motor fibres within these nerves have to be inhibited at some level in their path. Superior rectus is supplied by the superior division of the oculomotor nerve entering the inner surface of the muscle at the junction of its middle and posterior thirds. ${ }^{19}$ Inferior rectus, medial rectus, and inferior oblique are supplied by the inferior division of the oculomotor nerve entering the inner surface of the muscles at the junction of the middle and posterior thirds. Lateral rectus is supplied on its inner aspect by the abducens nerve just behind its middle. Only superior oblique is supplied superiorly at its lateral border by the trochlear nerve, the most anterior branch being at the junction of the posterior and middle thirds. All extraocular muscles, except superior oblique, are thus supplied by nerves entering on the inner surface. The presence of superior oblique function after effective blockade of all other extraocular muscle activity provides further evidence that a direct action upon nerve fibres in the retrobulbar compartment is the mechanism of action. To what degree the anaesthetic solution affects the ciliary ganglion is undetermined.

\section{Results}

Fifty consecutive patients who received local anaesthesia for cataract extraction had subTenon's anaesthesia and none experienced a problem of excessive discomfort on delivery of the block. Thirty four patients underwent extracapsular cataract extraction and 16 phakoemulsification, all with posterior chamber lens implantation. All local anaesthetic blocks were performed by the author and no complication occurred which prevented surgery. No failures in anaesthesia occurred which would have necessitated supplemental anaesthesia using 
Figure 4 Pain scores recorded for the delivery of the sub-Tenon local anaesthetic block. Eighteen patients recorded no pain or discomfort and a further 23 only slight discomfort.

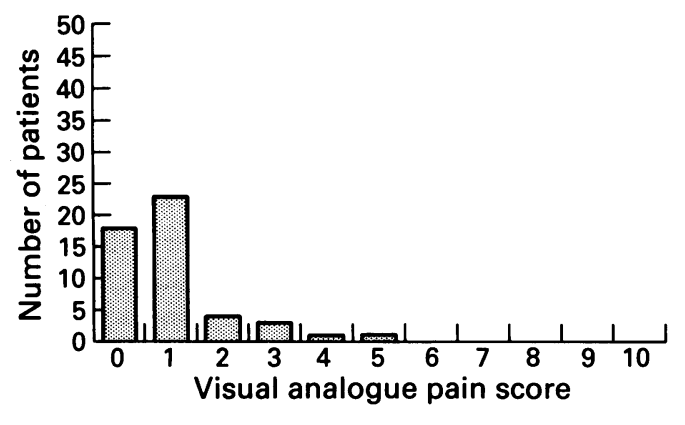

peribulbar infiltration. No patients who were scheduled for local anaesthesia were considered unsuitable for the one quadrant sub-Tenon's anaesthetic technique.

Pain scores assessed using the visual analogue score chart produced a median score of 1 ('slight discomfort') and mean of 1.0 for the delivery of the sub-Tenon's block (Fig 4). Eighteen patients graded no pain or discomfort and 23 only slight discomfort, with no patient grading greater than 5. Patients subjectively described the giving of the block most often as a feeling of 'slight pressure'.

For the subsequent operative procedure the median pain score was 0 ('no pain or discomfort') and the mean 0.3 (Fig 5); 41 patients scored 0 indicating the effectiveness of local anaesthesia felt during the operation. The one patient who gave a score of 7 ('severe pain') described the operation as pain free and would have scored it as 0 but felt sudden pain at the end of the operative procedure on giving subconjunctival injections. There was no difference of median pain scores between those having extracapsular extraction and phakoemulsification (median score of 0 ).

The anaesthetic block had no visible effect on superior oblique muscle function which remained for all 50 patients, though 27 patients developed otherwise complete akinesia. Those patients with incomplete akinesia most often had preserved lateral rectus muscle function. In patients where akinesia was not deemed sufficient by the surgeon (eight patients), a further 1-1.5 $\mathrm{ml}$ of solution was delivered to the original subTenon site. Excellent anaesthesia and good akinesia was then achieved within 5 minutes of delivery of the top-up and the operative procedure then commenced.

More conjunctival chemosis subjectively occurred than usually seen with peribulbar or retrobulbar anaesthesia and subconjunctival haemorrhage extending to more than one quadrant, assessed at the commencement of surgery, occurred in 16 patients $(32 \%)$. The use of adrenaline $0.1 \%$ immediately prior to the

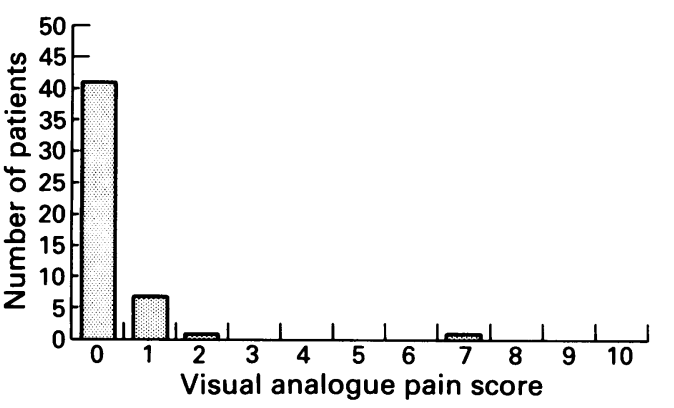



conjunctival incision is performed to attempt to reduce any subconjunctival haemorrhage by blanching conjunctival vessels. Complete ptosis occurred in 18 patients and partial ptosis in 15. Twenty three patients had an additional Van Lint facial block, subjectively based upon the presence of remaining eyelid tone after the subTenon block, immediately prior to surgery.

\section{Discussion}

The delivery of sub-Tenon's anaesthetic, directly irrigating the immediate retrobulbar region, is effective and reliable in providing both for anaesthesia and akinesia. Patients find not having an injection through skin, simple and relatively painless, except for those receiving a further facial block.

The method for sub-Tenon's irrigation described in this paper differs from that of Hanson et $a l,{ }^{6}$ in that anaesthetic is delivered to the inferior-nasal quadrant and no further opening of conjunctiva is performed. Hanson $e t$ al raise a conjunctival bleb by subconjunctival injection using a sharp needle, before incising superior conjunctiva, and then delivering subTenon solution. If limbal or scleral based cataract section is employed, superior quadrant application provides a simple method of delivery of solution peroperatively, though excess application of solution may result in a 'bulgy' eye if applied immediately prior to commencing intraocular surgery.

If solution is delivered to either of the superior quadrants possible chemosis and subconjunctival haemorrhage may affect visualisation of the operative section. Therefore it would be desirable to deliver anaesthetic to an inferior quadrant. The inferior temporal vortex vein emanates from the sclera $8 \mathrm{~mm}$ posterior to the inferior rectus muscle insertion at its temporal border. It then loops on the inner surface of Tenon's capsule and it may be damaged if entry into this quadrant is made without knowledge of the presence of the vein. The inferior-temporal quadrant is complicated by the presence of the inferior oblique muscle which passes up to run under lateral rectus. The inferior nasal quadrant also has a vortex vein but this is posteriorly situated and does not loop in the same way as the inferior temporal vortex vein. Entering conjunctiva inferiorly also avoids damage to superior conjunctiva, which is an important consideration if future glaucoma filtering surgery is to be performed, or a filtering bleb is already present. The inferior nasal quadrant has thus been chosen as the best quadrant to site delivery of anaesthesia, being the least likely to cause complication.

After delivery of the local anaesthetic solution surgery was performed no earlier than 15 minutes later, though anaesthesia has been described as occurring after only a few minutes using a different sub-Tenon's technique for panretinal photocoagulation. ${ }^{20}$ The 15 minute delay between delivery of block and commencement of surgery was to allow the solution time to diffuse, to achieve anaesthesia, akinesia, and to reduce any excess intraconal pressure on the globe. On application of the one quadrant block recorded no pain or discomfort, only one patient describing significant pain with a score of 7 . 
some proptosis is usually seen and the space occupying effect of the $3 \mathrm{ml}$ delivered would be expected to give some vis a tergo vitreous pressure which may give rise to a 'bulgy eye' if surgery were to begin immediately.

Possible problems associated with this inferior-nasal technique of anaesthesia include the requirement for the patient to elevate or abduct the eye to expose the inferior-nasal quadrant and allow the cannula to pass backward, though no patients during this study had any difficulty with this manoeuvre. Some peroperative chemosis is inherent with this technique and may be limited by ensuring posterior delivery of anaesthetic solution. Careful posterior delivery of anaesthetic not only prevents any chemosis extending to the region of the cataract section but also ensures that the block is truly delivered to the posterior subTenon's space and not inadvertently to the anterior subconjunctival space. An incision into conjunctiva and underlying fascia is made and a theoretical risk of providing a route for infection is possible, though any ocular surgery would normally have antibiotic cover by topical treatment which would also provide cover for this small incision.

Subconjunctival haemorrhage is caused by fine vessels which are inevitably severed on making the conjunctival cut. Though a visible haemorrhage may occur, it was often small and extended over one quadrant, though the opening of a subconjunctival route for haemorrhage to spread is facilitated by anteriorly leaking anaesthetic solution separating tissue planes. Though the haemorrhage was able to spread, it was in a thin layer and no patients had sufficient subconjunctival haemorrhage to cause a problem. Patients using anticoagulant medication, however, would require careful assessment before using the sub-Tenon technique due to the potential for haemorrhage from a severed conjunctival vessel. Use of the blunt cannula irrigation technique should, however, provide more safety, if local anaesthesia is used, than a sharp needle local anaesthesia technique.

One patient in this study had prior ocular surgery for retinal detachment. No difficulty was encountered with the delivery of the block except some Tenon's dissection was required to achieve a satisfactory sub-Tenon avenue for solution delivery. From the results of subTenon's anaesthesia in vitreoretinal surgery, the presence of scleral buckles, or previous surgery resulting in adhesions of Tenon's fascia, does not appear to present a problem with this form of block other than that extra dissection may be required.

Using a 50:50 mixture of lignocaine $2 \%$ and bupivacaine $0.5 \%$ the blocks have been found to have a rapid onset within a few minutes and to last a considerable length of time with some patients taking many hours to recover full ocular movements. The mean operative time for phakoemulsification was longer than for extracapsular extraction but the blocks remained effective for the duration of both procedures. In view of the efficacy of the block and the presence of ptosis, no additional facial block was used in 27 patients. To prevent risk of postoperative corneal exposure a dressing using a pad and covering shield were used routinely at the end of the procedure.

Anterior diffusion of solution to the subconjunctival space occurs by anterior solution delivery on forming the initial bleb and forward seepage of solution through the sub-Tenon path created by the infusion cannula. This gives rise to the observed subconjunctival chemosis. The conjunctival incision site provides a convenient, well-anaesthetised site to place a subconjunctival injection at the end of the operative procedure.

With familiarity the technique is relatively simple and quick to perform, offering excellent anaesthesia and akinesia, avoiding a sharp instrument being passed into the orbit. It provides a technique where the risk of accidental injection of solution to the subarachnoid space, into a blood vessel or of globe perforation should be practically eliminated, owing to use of the blunt cannula.

After over 100 years of sharp instrument local anaesthesia, a direct sub-Tenon irrigation approach, such as described here, could be viewed as returning full-circle to a modification of one of the first local anaesthetic techniques ever employed for ophthalmic surgery. ${ }^{2}$

The author acknowledges the assistance of $\mathrm{Mr}$ A M P Hamilton, Mr A D McG Steele of Moorfields Eye Hospital.

1 Knapp H. On cocaine and its use in ophthalmic surgery. Arch Ophthalmol 1884; 13: 402-48.

2 Turnbull CS. Editorial. Med Surg Rep 1884; Nov 29: 628 3 Ellis PR. Retrobulbar injections. Surv Ophthalmol 1974; 18: 425-30.

4 Bloomberg LB. Administration of periocular anaesthesia. $f$ Cataract Refract Surg 1980; 12: 677-9.

5 Smith R. Cataract extraction without retrobulbar anaesthetic injection. Br f Ophthalmol 1990; 74: 205-7.

6 Hansen EA, Mein CE, Mazzoli R. Ocular anesthesia for cataract surgery: a direct sub-tenon's approach. Ophthalmic Surg 1990; 21: 696-9.

7 Morgan CM, Schatz H, Vine AK, Cantrill H, Davidorf FH, Gitter KA, et al. Ocular complications associated with retrobulbar injections. Ophthalmology 1988; 95: 660-5.

8 Smith JL. Retrobulbar bupivacaine can cause respiratory arrest. Ann Ophthalmol 1982; 14: 1005-6.

9 Kimble JA, Morris RE, Witherspoon CD, Feist RM. Globe perforation from peribulbar injection. Arch Ophthalmol 1987; 105: 749.

10 Hay A, Flynn HW Jr, Hoffman JI, Rivera AH. Needle penetration of the globe during retrobulbar and peribulbar penetration of the globe during retrobulbar
injections. Ophthalmology 1991; 98: 1017-24.

11 Redmond RM, Dallas NL. Extracapsular cataract extraction under local anaesthesia without retrobulbar injection. $\mathrm{Br} \mathcal{F}$ Ophthalmol 1990; 74: 203-4.

12 Petersen WC, Yanoff M. Subconjunctival anaesthesia: an alternative to retrobulbar and peribulbar techniques. Ophthalmic Surg 1991; 22: 199-201.

13 Furuta M, Toriumi T, Kashiwagi K, Satoh S. Limbal anesthesia for cataract surgery. Ophthalmic Surg 1990; 21: 22-5.

$14 \mathrm{Khoo}$ CY. Local anaesthesia without retrobulbar injection. $\mathrm{Br}$ f Ophthalmol 1990; 74: 639 .

15 Mein CE, Woodcock MG. Local anesthesia for vitreoretinal surgery. Retina 1990; 10: 47-9.

16 Parks MM. Extraocular muscles. In: Duane TD, Jaeger EA, eds. Clinical ophthalmology. Philadelphia: Harper \& Row, 1987: Vol 1, Ch 1

17 Koorneef $L$. New insights in the human orbital connective tissue. Result of a new anatomical approach. Arch Ophthalmol 1977; 95: 1269-73.

18 Abelson MB, Mandel E, Paradis A, George M. The effect of hyaluronidase on akinesia during cataract surgery. Ophthalmic Surg 1989; 20: 325-6.

19 Eugene Wolff's anatomy of the eje and orbit. 7th ed revised by Warwick R, London: H K Lewis, 1976: 309-13.

20 Friedberg M, Palmer RM. A new technique of local anesthesia for panretinal photocoagulation. Ophthalmic Surg 1991; 22: 619-21. 